Classification

Physics Abstracts

$05.40-05.50-66.30$

\title{
The fractal nature of a diffusion front and the relation to percolation
}

\author{
B. Sapoval, M. Rosso and J. F. Gouyet \\ Laboratoire de Physique de la Matière Condensée $\left({ }^{*}\right)$, Ecole Polytechnique, 91128 Palaiseau, France
}

(Reçu le 30 octobre 1984, accepté le 20 décembre 1984)

\begin{abstract}
Résumé. - On montre sur une simulation à deux dimensions qu'un front de diffusion possède une géométrie fractale sur une largeur dépendant de la longueur de diffusion. Le nombre de particules sur le front, et la largeur mesurant son étalement, suivent des lois de puissance en fonction de la longueur de diffusion. Les exposants de ces lois et la dimension fractale peuvent être écrits simplement en fonction des exposants critiques de la percolation à deux dimensions.
\end{abstract}

\begin{abstract}
Using a two dimensional simulation, a diffusion front is shown to have a fractal geometry in a range increasing with the diffusion length. The number of particles on the front, and the width measuring its spread, follow power laws as a function of the diffusion length. The associated exponents and the fractal dimension can be expressed as simple functions of the critical exponents of the two dimensional percolation problem.
\end{abstract}

In the simplest picture, diffusion in solids is the consequence of Brownian motion of atoms on a lattice. Brownian motion is known to create fractal patterns [1,2]. The purpose of this paper is to show, through a numerical simulation on a $2 \mathrm{D}$ lattice, that the diffusion front is a fractal object [3] with dimension $D_{\mathrm{f}}=1.76 \pm 0.02$. Moreover we put forward the idea that there is a close similarity between this line and the external frontier of a percolating cluster. These considerations may have practical importance since, for example, atomic diffusion plays a role in many ways in realizing electrical contacts : a good contact between two bodies can often be realized by heating the system so enabling diffusion to occur in a reasonably short time.

We consider the simple case of the diffusion of particles $A$ on a 2D lattice $B$ with the diffusion source being a line of $\mathrm{A}$ atoms kept at a constant concentration equal to unity. We restrict the analysis to nearest neighbour site hopping without double occupancy. Under these circumstances the concentration varies as a function of time and space as [4]

$$
p(x)=\operatorname{erfc}\left(x / l_{D}\right)=1-\left(2 / \pi^{1 / 2}\right) \int_{0}^{x / l_{D}} \mathrm{~d} u \exp \left(-u^{2}\right)
$$

where $l_{D}=2(D t)^{1 / 2}$ is the diffusion length at time $t$ with diffusion coefficient $D$. The average

(*) Groupe de Recherche du CNRS. 
concentration is a function of time and space but in the following we will only consider the situations obtained at fixed successive times. Both square and triangular lattices will be investigated.

We define the microscopic physical interface in a simple manner which is illustrated by the following electrical picture. The diffusing A atoms are supposed to be metallic, and " conducting " only if they are nearest neighbours, the B lattice being otherwise insulating. In this picture if a potential is applied between materials A and B all the occupied sites which are connected with $A$ are at the same electrical potential. We investigate the frontier of this region. In the case of a square lattice the frontier is made of those A atoms which are connected through nearest neighbours with the diffusion source and have an empty first or second neighbour which itself is connected through empty first or second neighbours to the region far in B where no A atom are present. This is shown in figure 1 where we see that occupied sites build a land connected to the diffusion source, and also islands. The empty sites create an " open sea " and lakes. The object we are looking at is the thick line in figure $1:$ it is the " seashore ». Correlations between particles may play a role in the structure of this seashore. In this simplified model we neglect such effects : a given diffused state is simply obtained by a random distribution of particles obeying equation (1). This procedure provides for a considerable gain in computing time compared to the complete simulation of diffusion.

The simulation is made by choosing for each site in a row of atoms at constant $x$ a random number between 0 and 1 . If this random number is smaller than $p(x)$ as given by equation (1), we put an atom in that site. In this way we constitute one detailed diffusion picture on which we look for the seashore as defined above. Periodic boundary conditions are used to define this seashore. We name $p_{\mathrm{f}}(x)$ the probability of finding one point of the frontier at $x$. It is the density of seashore at distance $x$. This quantity is obtained from the simulation calculation. It is found to behave very nearly as a Gaussian function of $x$. These density profiles are then averaged over 100 different diffusion pictures of lateral size $L=512$ or $L=1024$ (see Fig. 2a)

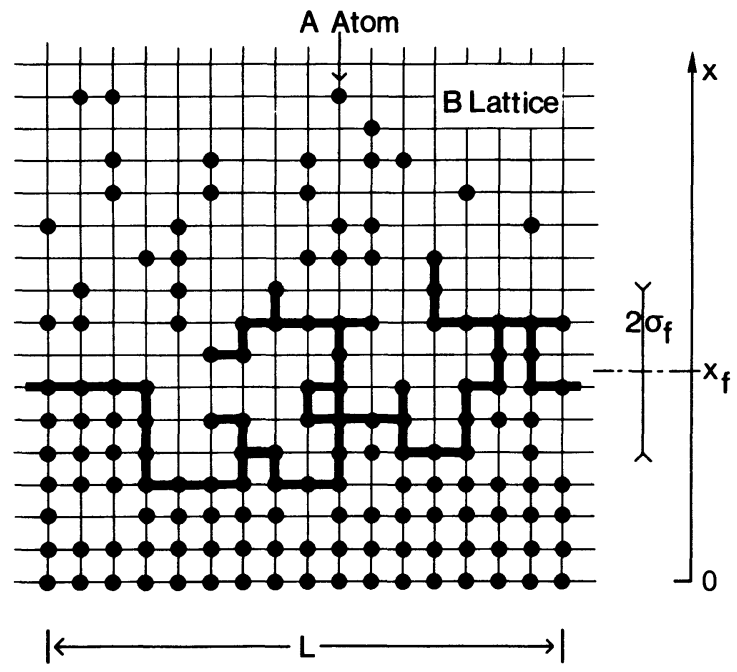

Fig. 1. - Schematic picture of the simulated diffused system at a given time. The source of $A$ atoms at the bottom is kept at unit concentration. The concentration decreases with increasing $x$. The thick line represents the diffusion front that we are studying. Its mean position is $x_{\mathrm{f}}$ and it is spread on a width $\sigma_{\mathrm{f}}$. 


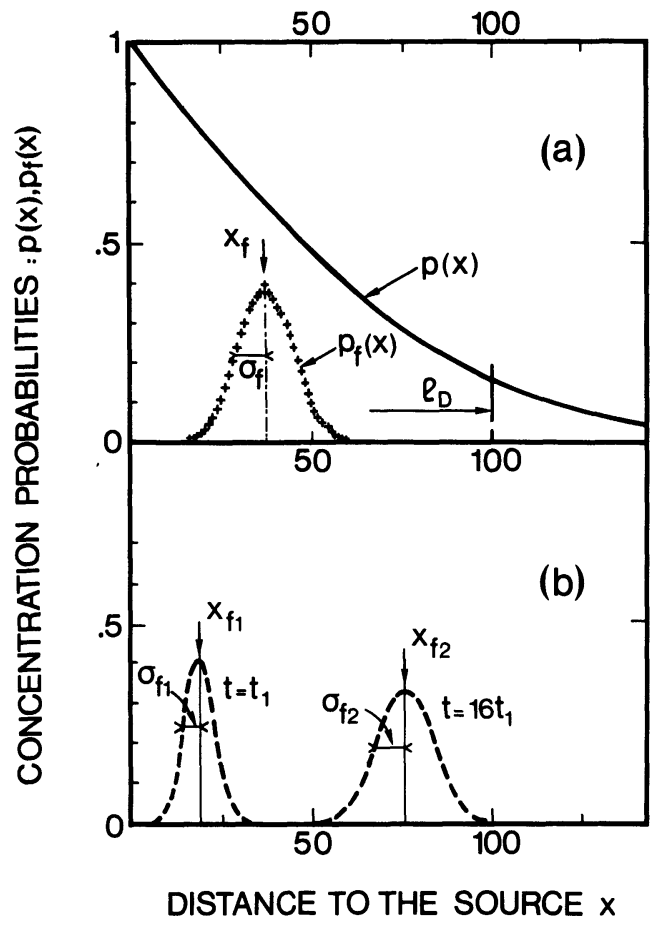

Fig. 2. - Statistical distribution of the simulated frontier defined in figure 1 in the case of a square lattice. (a) Comparison of the frontier density (crosses) with the overall atom density (continuous line) at a given time. (b) Frontiers for $l_{D, 1}=50$ and $l_{D, 2}=200$.

and we calculate the mean position $x_{\mathrm{f}}$ and the width $\sigma_{\mathrm{f}}$ using

$$
x_{\mathrm{f}}=\int_{0}^{\infty} x p_{\mathrm{f}}(x) \mathrm{d} x / \int_{0}^{\infty} p_{\mathrm{f}}(x) \mathrm{d} x
$$

and

$$
\sigma_{\mathrm{f}}^{2}=\int_{0}^{\infty}\left(x-x_{\mathrm{f}}\right)^{2} p_{\mathrm{f}}(x) \mathrm{d} x / \int_{0}^{\infty} p_{\mathrm{f}}(x) \mathrm{d} x .
$$

The results are qualitatively represented in figure $2 \mathrm{~b}$. One can see two different profiles of the frontier for two different times corresponding to $l_{D, 1}=50$ and $l_{D, 2}=200$. The mean position $x_{\mathrm{f}}$ varies like $l_{D}$. Figure 3 gives the quantitative results for the width $\sigma_{\mathrm{f}}$ and the number of points on the frontier $N_{\mathrm{f}}$. We observe that these quantities follow power laws to within a very good approximation. This is discussed below.

The geometry of the frontier has been determined by the mass to radius relation [1] :

$$
M \sim R^{D_{\mathrm{f}}} .
$$

In our case we take as the mass the number of sites of the frontier in a square of side $R$. Figure 4 shows the log-log plot in two cases with two very different values of the diffusion length. We observe that the fractal geometry exists only up to a scale of the order of $\sigma_{\mathrm{f}}$. Because of the dependence of $\sigma_{\mathrm{f}}$ on $l_{D}$ the fractal geometry exists on a scale which increases with the diffusion length. 


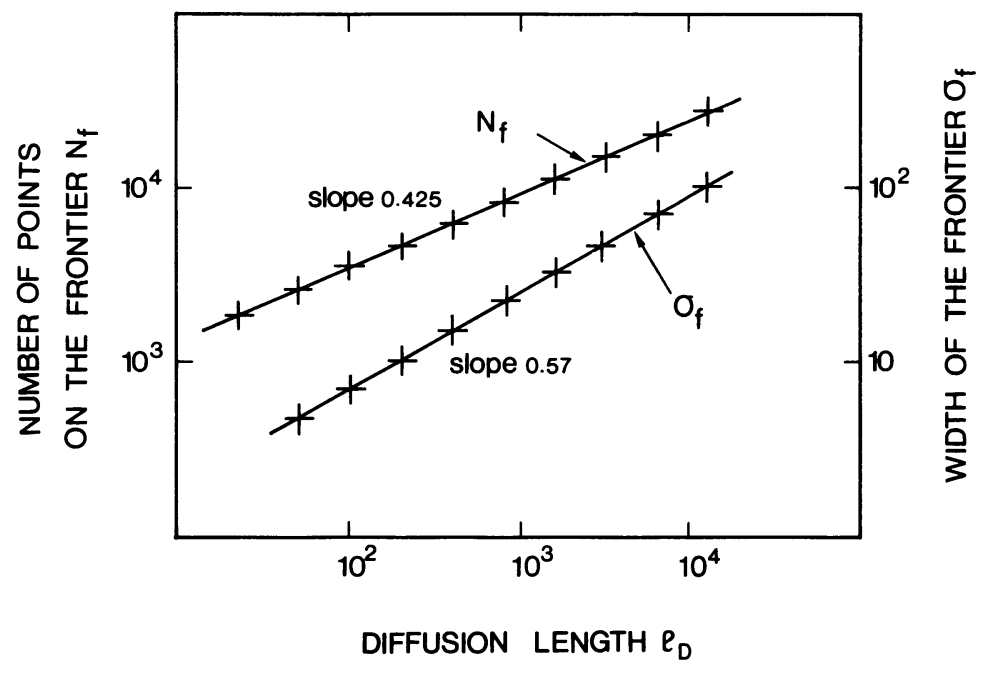

Fig. 3. - Variation of the number of points of the frontier $N_{\mathrm{f}}$ and of the width $\sigma_{\mathrm{f}}$ as a function of the diffusion length $l_{D}$. The above results for $N_{\mathrm{f}}$ have been obtained for, or rescaled to, a sample lateral size of $L=512$ in the case of the square lattice.

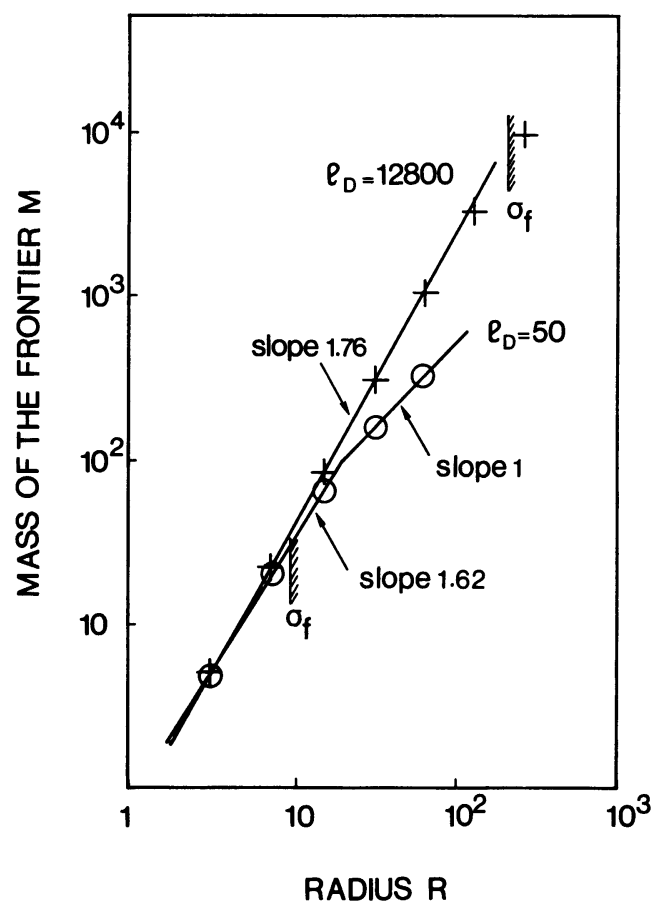

Fig. 4. - Determination of the fractal dimension of the diffusion front from the mass-to-radius relation (square lattice). The diffusion front is a fractal only up to a scale of the order of the width $\sigma_{\mathrm{f}}$. The value 1.62 quoted for $l_{D}=50$ comes from a finite size effect which disappears for $l_{D} \geqslant 1600$. 
The dimension is one when $R$ is larger than a few $\sigma_{\mathrm{f}}$. This only tells us that the length of the frontier is proportional to the lateral size $L$ of the sample. ( $L$ is always taken larger than $\sigma_{\mathrm{fr}}$ ) We obtain in the fractal region :

$$
D_{\mathrm{f}}=1.76 \pm 0.02 \text {. }
$$

We want now to show that the above physical situation is closely related to the usual percolation problem. The percolation problem is defined with a constant concentration probability over the lattice. One generally thinks that because percolation is a critical phenomenon the probability of occupation should be kept rigorously constant, otherwise the critical effects are expected to be smeared out. In fact this is not true and interesting new features appear in the present situation.

Consider the diffusion system defined above at a given time : it is a random distribution but with non-uniform probability. There is obviously an infinite cluster which links both lateral sides of the sample because the concentration near the source is close to unity. Consider now the geometry of this cluster and its penetration depth. Here again it is obvious that far from the source the infinite cluster has no branches because in these regions the concentration is too small. Hence the frontier of the infinite cluster should be in a region where the concentration is around the critical concentration $p_{\mathrm{c}}$ for the considered lattice. The frontier has a mean distance to the source $x_{\mathrm{f}}$ and thus we expect $p\left(x_{\mathrm{f}}\right)$ to be near $p_{\mathrm{c}}$. The result of the simulation is shown in table I for the two cases of the square and triangular lattices. A striking result is obtained : the simulated frontier is situated at $p_{\mathrm{c}}$. More precisely it appears that the concentration at $x_{\mathrm{f}}$ tends asymptotically towards $p_{\mathrm{c}}$ in the infinite diffusion limit. But already for $l_{D}=100$ the deviation is only $1 \%$, both for square and triangular lattices. For $l_{D}=6400$ this deviation is as small as $0.2 \%$.

This explains a result previously mentioned : because $x_{\mathrm{f}}$ appears to correspond to a constant concentration it varies proportionally to the diffusion length $l_{D}$. This is a consequence of equation (1) which indicates that a given $p$ is obtained for a constant value of $x_{\mathrm{f}} / l_{D}$. Values of $x_{\mathrm{f}} / l_{D}$ are shown in table I for various choices of $l_{D}$ for square and triangular lattices. If we postulate that

$$
p\left(x_{\mathrm{f}}\right)=p_{\mathrm{c}}
$$

we expect $x_{\mathrm{f}}=0.4769 l_{D}$ on the triangular lattice where $p_{\mathrm{c}}=0.5$ and $x_{\mathrm{f}}=0.378 l_{D}$ on the square lattice using $p_{\mathrm{c}}=0.593$ [5]. One sees in table I that the results of the simulation on finite samples are very close to these values.

Table I. - Mean position of the frontier and concentration of diffused atoms at this position. These results are obtained from the 2D simulation for selected values of the diffusion length, both for square and triangular lattices. The values of $p_{\mathrm{c}}$ for these two lattices are respectively 0.593 and 0.5 [7].

\begin{tabular}{|c|c|c|c|c|}
\hline & $l_{D}$ & 100 & 800 & 6400 \\
\hline \multirow{3}{*}{ Square lattice } & $x_{\mathrm{f}} / l_{\boldsymbol{D}}$ & $0.3736 \pm 0.0008$ & $0.3771 \pm 0.0006$ & $0.3781 \pm 0.0003$ \\
\cline { 2 - 5 } & $p\left(x_{\mathrm{f}}\right)$ & $0.5973 \pm 0.0008$ & $0.5938 \pm 0.0006$ & $0.5929 \pm 0.0003$ \\
\hline \multirow{3}{*}{ Triangular lattice } & $x_{\mathrm{f}} / l_{\boldsymbol{D}}$ & $0.4699 \pm 0.0008$ & $0.4755 \pm 0.0006$ & $0.4757 \pm 0.0003$ \\
\cline { 2 - 5 } & $p\left(x_{\mathrm{f}}\right)$ & $0.506 \pm 0.0007$ & $0.5013 \pm 0.0006$ & $0.5011 \pm 0.0003$ \\
\hline
\end{tabular}


Moreover the external frontier of the percolating cluster, the so-called " hull », has in fact the same definition as our seashore [6]. Thus the above ideas suggest that the diffusion front should have the same geometric properties as the percolation hull. The fractal dimension of the percolation hull has been determined for the square lattice to be $D_{\mathrm{h}}=1.74 \pm 0.02$ or $D_{\mathrm{h}}=$ $1.76 \pm 0.01$ [6]. Comparison with the value $D_{\mathrm{f}}=1.76 \pm 0.02$ that we have determined supports the idea of a direct similarity between the diffusion front and the percolation hull.

We then start from the same consideration to predict theoretically the exponents for the dependence on $l_{D}$ of the width $\sigma_{\mathrm{f}}$ and the length $N_{\mathrm{f}}$ of the diffusion front. From the data shown in figure 4 we obtain

$$
\begin{aligned}
\sigma_{\mathrm{f}} & =0.46\left(l_{D}\right)^{\alpha_{\sigma}} \text { with } \alpha_{\sigma}=0.57 \pm 0.01 \\
N_{\mathrm{f}} & =L \int_{0}^{\infty} p_{\mathrm{f}}(x) \mathrm{d} x \\
& =0.96 L\left(l_{D}\right)^{\alpha_{N}} \text { with } \alpha_{N}=0.424 .
\end{aligned}
$$

When $x$ is larger or smaller than $x_{\mathrm{f}}$ the concentration deviates from $p_{\mathrm{c}}$ due to the gradient of the particle concentration. There are lakes and islands in these regions. The characteristic size of these objects should be of the order of the correlation length [7] corresponding to the value of the concentration at that coordinate :

$$
\xi(x)=\xi_{0}\left|p(x)-p_{c}\right|^{-v}
$$

where $v$ is the usual critical exponent and $\xi_{0}$ is of the order of the lattice parameter [7].

If the distance to $x_{\mathrm{f}}$ of a cluster (lake or island) of characteristic size $\xi(x)$ is equal to $\xi$ this cluster has obviously a finite probability of touching the frontier and thus to be within the frontier width. In other words

$$
\sigma_{\mathrm{f}}=K \xi\left(x_{\mathrm{f}} \pm \sigma_{\mathrm{f}}\right)
$$

where $K$ should be of the order of unity.

Using equations (1), (6), (7) and (9) we derive from (10) by a Taylor expansion

$$
\begin{aligned}
\sigma_{\mathrm{f}} & =K \xi_{0}\left|\sigma_{\mathrm{f}}\left(\frac{\partial p}{\partial x}\right)_{x=x_{\mathrm{f}}}\right|^{-v} \\
& =K \xi_{0}\left(0.977 \frac{\sigma_{\mathrm{f}}}{l_{\mathrm{D}}}\right)^{-v}
\end{aligned}
$$

thus

$$
\sigma_{\mathrm{f}}=K^{\prime} \xi_{0}^{\frac{1}{1+v}} \frac{v}{l_{D}^{1+v}}
$$

where $K^{\prime}$ is also of the order of one. Then we find

$$
\alpha_{\sigma}=\frac{v}{1+v} .
$$

If we use for $v$ the value [8] $v=4 / 3$ we predict $\alpha_{\sigma}=4 / 7=0.5714$, while the simulation gives $\alpha_{\sigma}=0.57 \pm 0.01$. The value of the prefactor obtained from the simulation (relation (7)) indicates that equation (10) is well verified.

We want now to interpret the exponent $\alpha_{N}$ that we observe for the dependence of $N_{\mathrm{f}}$ on $l_{D}$ (Fig. 4). In fact $N_{\mathrm{f}}$ is not an intrinsic quantity because it is proportional to the lateral size $L$ of the sample for $L$ larger than $\sigma_{\mathrm{f}}$. We propose to consider a more intrinsic quantity $N_{\mathrm{f}} \sigma_{\mathrm{f}} / L$ which 
is the number of points on the frontier on a lateral scale $\sigma_{\mathrm{f}}$. From (8) and (11) one can write

$$
\frac{N_{\mathrm{f}} \sigma_{\mathrm{f}}}{L} \sim l_{D}^{\alpha_{N}} \frac{v}{l_{D}^{1+v}}
$$

But as shown above the frontier is a fractal on this scale so that from equations (4) and (11)

$$
\frac{N_{\mathrm{f}} \sigma_{\mathrm{f}}}{L} \sim \sigma_{\mathrm{f}}^{D_{\mathrm{f}}} \sim l_{D}^{\frac{r}{1+v} D_{\mathrm{f}}}
$$

By comparing (13) and (14) one finds

$$
\alpha_{N}=\frac{v}{1+v}\left(D_{\mathrm{f}}-1\right)
$$

Using $v=4 / 3$ and our measured value $D_{\mathrm{f}}=1.76 \pm 0.02$ we predict $\alpha_{N}=0.43 \pm 0.01$ while the simulation gives $\alpha_{N}=0.425 \pm 0.005$.

A remarkable feature arises from table II where the values of $\left(N_{\mathrm{f}} \sigma_{\mathrm{f}}\right) /\left(L l_{D}\right)$ are shown. This last

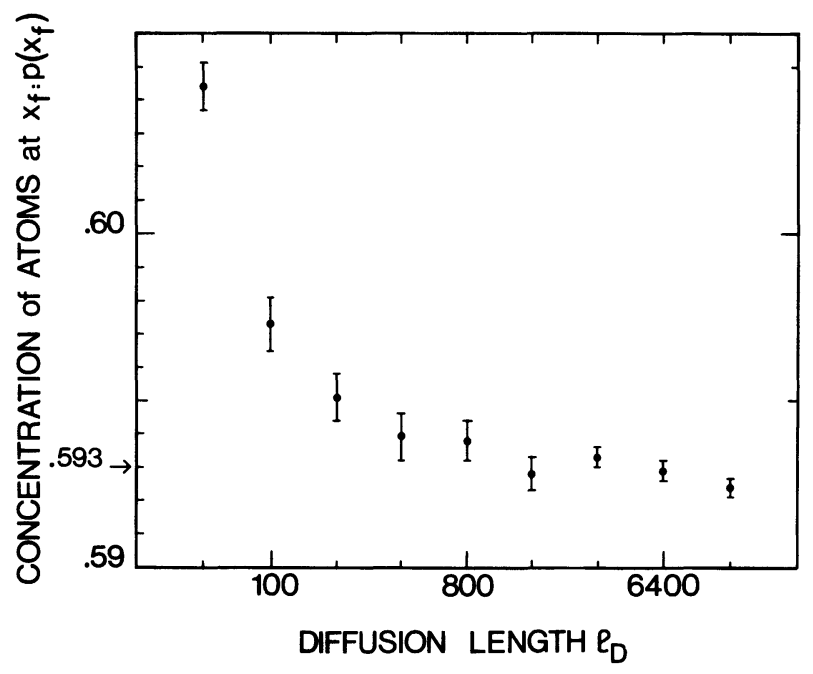

Fig. 5. - Diffused atom concentration at the mean front position $x_{\mathrm{f}}$ as a function of the diffusion length in the case of a square lattice. The approximate value $p_{c}=0.593$ has been represented. The error bars represent statistical uncertainties.

Table II. - Values of $N_{\mathrm{f}} \sigma_{\mathrm{f}} / L l_{D}$. The result of the simulation suggests that this quantity tends to a constant value when $l_{D}$ goes to infinity.

\begin{tabular}{|c|c|c|c|c|}
\hline$l_{D}$ & 200 & 800 & 1600 & 6400 \\
\hline$\frac{N_{\mathrm{f}} \sigma_{\mathrm{f}}}{L l_{D}}$ & 0.464 & 0.447 & 0.442 & 0.441 \\
\hline
\end{tabular}


quantity should vary as

$$
\frac{N_{\mathrm{f}} \sigma_{\mathrm{f}}}{L l_{D}} \sim l_{D}^{\left(\alpha_{N}+\alpha_{\sigma}-1\right)} .
$$

In table II we see that this quantity is almost constant indicating that the number of points of the frontier contained in a square of side equal to the width of the frontier is proportional to the diffusion length, hence to the total number of diffused atoms. The exponent appearing in (16) should be zero, thus $\alpha_{N}+\alpha_{\sigma}-1=0$ and

$$
\alpha_{N}=\frac{1}{1+v}=3 / 7=0.4286
$$

Moreover from(12) and (15) we conjecture that

$$
D_{\mathrm{f}}=\frac{1+v}{v}=7 / 4=1.75
$$

may hold in two dimensions. The same relation should hold for $D_{h}$ the fractal dimension of the percolation hull.

In conclusion we have shown that the diffusion front is a fractal line with the same dimension as the " hull " of the infinite cluster in the percolation problem on a $2 \mathrm{D}$ lattice. The observation that the mean frontier occurs in the region where the concentration of diffused atoms is equal to the percolation value $p_{c}$ indicates the close connection between this problem and the percolation problem. Moreover the width and the number of points on the frontier depends on the diffusion length through power laws, whose exponents are obtained with a very good precision. These exponents are not critical exponents but appear to be simply related to the critical exponents of percolation. This result suggests that a fruitful approach to percolation problems can be obtained in systems with a gradient of concentration. The case of a linear gradient will be discussed in detail in a forthcoming paper [9].

\section{References}

[1] Mandelbrot, B. B., The fractal geometry of nature (W. H. Freeman and Company, San Francisco) 1982 and references therein.

[2] Witten, JR., T. A. and SANDER, L. M., Phys. Rev. Lett. 47 (1981) 1400.

[3] This work was presented at the CECAM workshop on " Kinetic models for cluster formation " held at Orsay (France, September 1984). Preliminary results on diffusion were presented at the workshop on " Fractals in Physics » held at Les Houches (France, March 1984).

[4] Dieterich, W., Fulde, P. and Peschel, I., Adv. Phys. 29 (1980) 527.

[5] Djordjevic, Z. V., Stanley, H. E. and Margolina, A., J. Phys. A 15 (1982) L405;

Derrida, B. and De Seze, L., J. Physique 43 (1982) 457 ;

Gebele, T., J. Phys. A 17 (1984) L51.

[6] Voss, R. F., J. Phys. A 17 (1984) L373.

[7] Stauffer, D., Phys. Rep. 54 (1979) 1.

[8] Riedel, E. K., Physica A 106 (1981) 110.

[9] Rosso, M., GouYet, J. F. and SaPOVal, B., to be published. 